\title{
Composición nutricional de seis gramíneas tropicales del estado de Jalisco, México
}

\section{Nutritional composition of six tropical grasses from the state of Jalisco, Mexico}

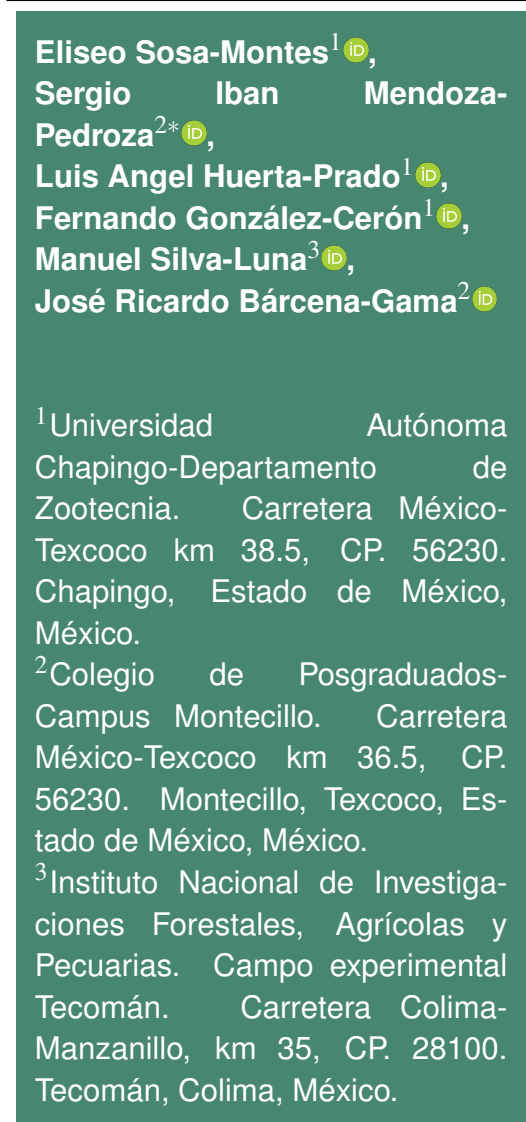

*Autor de correspondencia: sergiomp@colpos.mx

\section{Artículo científico}

Recibido: 02 de marzo 2021 Aceptado: 02 de marzo 2021

Como citar: Sosa-Montes E, Mendoza-Pedroza SI, HuertaPrado LA, González-Cerón F, Silva-Luna M, Bárcena-Gama JR (2021) Composición nutricional de seis gramíneas tropicales del estado de Jalisco, México. Ecosistemas y Recursos Agropecuarios Núm. Esp. II: e2938. DOI:
RESUMEN. El objetivo de este estudio fue evaluar la composición química de seis gramíneas forrajeras para el trópico húmedo y seco mexicano: Andropogon gayanus, Andropogon gerardii, Cenchrus ciliaris, Chloris gayana, Megathyrsus maximus y Urochloa brizantha. Las muestras se colectaron en noviembre de 2019, en Tonaya, Jalisco, México. Se determinaron: materia seca, cenizas, proteína cruda (total e insoluble), extracto etéreo, fibra detergente neutro, fibra detergente ácido, hemicelulosa, lignina y celulosa. La evaluación se realizó en el Laboratorio de Nutrición Animal del Departamento de Zootecnia, Universidad Autónoma Chapingo, Texcoco, Estado de México. Cada gramínea se evaluó por triplicado y cada repetición se consideró como unidad experimental. Las medias de composición química fueron separadas mediante la prueba de Tukey $(p<0.05)$. La correlación entre cada par de variables $(p<0.05)$ se estimó mediante el coeficiente de Pearson $(p<0.05)$. A mayor porcentaje de proteína total $(p<0.05)$ menor porcentaje de extracto etéreo y mayores porcentajes de cenizas y de proteína insoluble. Además, a mayor porcentaje de proteína insoluble, menor porcentaje de lignina $(p<0.05)$. Probablemente, la proteína se concentra en su fracción insoluble a expensas del extracto etéreo y la lignina. Consecuentemente, gran-tallo-azul (Andropogon gerardii) mostró porcentajes altos de proteína total $(10.27 \%)$ y cenizas $(7.88 \%)$, y bajos de lignina $(5.23 \%)$ y extracto etéreo $(1.37 \%)$; pero su proteína insoluble no fue tan elevada (2.06\%) como la de guinea (Megathyrsus maximus) $(6.08 \%)$. Por tanto, A. gerardii tuvo el mejor contenido nutricional a los 35 días de rebrote.

Palabras clave: Composición química, forrajes para el trópico mexicano, proteína cruda en pastos tropicales.

ABSTRACT. The objective of this study was to evaluate the chemical composition of six forage grasses for the Mexican wet and dry tropics: Andropogon gayanus, Andropogon gerardii, Cenchrus ciliaris, Chloris gayana, Megathyrsus maximus and Urochloa brizantha. Samples were collected on November 2019, in Tonaya, Jalisco, Mexico. Dry matter, ashes, crude protein (total and insoluble), ether extract, neutral detergent fiber, acid detergent fiber, hemicellulose, lignin and cellulose were determined. The evaluation was carried out at the Animal Nutrition Laboratory, Animal Science Department, Chapingo Autonomous University, Texcoco, State of Mexico. Each grass was evaluated, in triplicate, and each replicate was considered the experimental unit. Means of chemical composition were separated by the Tukey test $(p<0.05)$. The correlation between each pair of variables was estimated by the Pearson coefficient $(p<0.05)$. The higher the percentage of total protein $(p<0.05)$, the lower the percentage of ether extract and the higher the percentages of ashes and insoluble protein. Furthermore, the higher the percentage of insoluble protein, the lower the percentage of lignin $(p<0.05)$. Probably, protein concentrates in its insoluble fraction at the expense of ether extract and lignin. Consequently, great-blue-stem (Andropogon gerardii) showed high total protein $(10.27 \%)$ and ashes $(7.88 \%)$, and low percentages of lignin $(5.23 \%)$ and ether extract $(1.37 \%)$; but its insoluble protein was not as high $(2.06 \%)$ as that of guinea grass (Megathyrsus maximus) (6.08\%). Therefore, great-blue-stem had the best nutritional content at 35 días of regrowth.

Key words: Chemical composition, forages for the Mexican tropics, crude protein in tropical grasses. 


\section{INTRODUCCIÓN}

México ocupa el octavo lugar a nivel mundial en población de ganado bovino, con un inventario de 31 millones de cabezas $(2.31 \%$ del hato mundial). Dicha población ganadera se mantiene en una superficie de 147 millones de hectáreas, lo que representa el $73 \%$ de la superficie del territorio nacional (Enríquez et al. 2011). Los sistemas de producción pecuaria se han tenido que adecuar a diversos ecosistemas en México, ya que, cuenta con diferentes tipos de clima, clases de suelo, tipos de vegetación, y características fisiográficas. Por ello, se ha subdividido al territorio nacional en cinco grandes zonas ecológico-ganaderas; árida, semiárida, templada, trópico seco y trópico húmedo (Villegas et al. 2001). La producción animal en las regiones tropicales de México se desarrolla principalmente bajo condiciones de pastoreo extensivo con gramas nativas, este sistema de producción se caracteriza por bajos costos, pero en consecuencia la productividad del ganado es baja. Esto hace necesario hacer ganadería intensiva con un uso rentable y eficiente de los recursos naturales disponibles, los forrajes (Peña y Del Pozo 2004). Distintos factores como la edad pueden hacer que la proteína cruda de las gramíneas sea baja y con mucha pared celular o fibra detergente neutro, lo que las hará menos digestibles (Castrejón et al. 2017). Por ejemplo, la proteína cruda disminuye $7 \%$ en gramas nativas respecto a su asociación con una gramínea mejorada como Arachis pintoi y varía entre especies de pastos (Fernández et al. 2006).

Por ello, en el Rancho San Luis Tenango en el Municipio de Tonaya, Jalisco, México se colectaron las siguientes especies de gramíneas que se describen brevemente a continuación: Andropogon gayanus Kunth (pasto llanero), originario de África, puede crecer hasta $400 \mathrm{~cm}$, a las seis semanas del rebrote contiene: $6.2 \%$ proteína, $7.6 \%$ cenizas, $72.1 \%$ fibra detergente neutro y $43.2 \%$ fibra detergente ácido (Castrejón et al. 2017); Andropogon gerardii Vitman (gran-tallo-azul o pie de pavo), originario de América del norte, cosechada a las 12 semanas de rebrote produjo: $5.5 \%$ proteína (Kupryś-
Caruk et al. 2019). Cenchrus ciliaris L. (pasto buffel), originario de Asia y África, tiene alturas de entre $30 \mathrm{y}$ $200 \mathrm{~cm}$ en madurez, a las ocho semanas del rebrote contiene: $4.6 \%$ proteína, $10.2 \%$ cenizas, $70.5 \%$ fibra detergente neutro y $52.4 \%$ fibra detergente ácido (Castrejón et al. 2017). Chloris gayana Kunth (pasto rhodes), originario de África, alturas de más de 100 $\mathrm{cm}$ en su madurez, a las 11 semanas de edad produjo aproximadamente: $5.5 \%$ proteína, $14 \%$ cenizas y 73\% fibra detergente neutro (Daba et al. 2019). Megathyrsus maximus (Jacq.) B.K. Simon \& S.W.L. Jacobs (pasto guinea), originario de África y Asia, en época de lluvias florece a 45 días después del rebrote y tiene alturas hasta de $350 \mathrm{~cm}$, a las seis semanas del rebrote contiene: $5.7 \%$ proteína, $11.9 \%$ cenizas, $71.5 \%$ fibra detergente neutro y $45 \%$ fibra detergente ácido. Urochloa brizantha (Hochst. Ex A. Rich.) R.D. Webster (pasto insurgente), originario de África subsahariana, tiene alturas hasta de 200 $\mathrm{cm}$, a las cinco semanas de rebrote contiene: $7.3 \%$ proteína, $11.9 \%$ cenizas, $67.1 \%$ fibra detergente neutro y $46.6 \%$ fibra detergente ácido (Castrejón et al. 2017). En la literatura se menciona que las distintas gramíneas varían en composición química. Por ello en el presente trabajo el objetivo fue conocer la calidad nutricional en época de lluvias de seis gramíneas que se colectaron en el municipio de Tonaya, Jalisco para determinar cuál de ellas puede ser la mejor opción en la alimentación del ganado.

\section{MATERIALES Y MÉTODOS}

\section{Localidad}

El trabajo se realizó durante el periodo de lluvias (noviembre de 2019), en el Rancho San Luis Tenango en el Municipio de Tonaya, Jalisco, México entre las coordenadas $19^{\circ} 51^{\prime} 10.78^{\prime \prime}$ LN y $103^{\circ} 58^{\prime}$ 6.48 " LO, a $800 \mathrm{msnm}$. En el sitio se distinguieron dos tipos de clima: cálido-subhúmedo y semicálidosemihúmedo, que corresponden al 57.6 y $42.4 \%$ de la superficie, respectivamente. La temperatura media anual es de $22.4{ }^{\circ} \mathrm{C}$, con máxima promedio de 33.4 ${ }^{\circ} \mathrm{C}$ y mínima promedio de $10.9{ }^{\circ} \mathrm{C}$. La precipitación media anual es de 800 mm (IIEG-Jalisco 2018). 


\section{Colecta de muestras}

El día 15 de noviembre de 2019, aproximadamente a las 5 semanas de rebrote, los pastos $A n$ dropogon gayanus, Andropogon gerardii, Cenchrus ciliaris, Chloris gayana, Megathyrsus maximus y Urochloa brizantha, se seleccionaron de forma aleatoria, de forma manual cosechar el forraje y colocarlo en bolsas de papel previamente rotuladas con tres repeticiones. Posteriormente, con ayuda de una estufa de aire forzado a $55^{\circ} \mathrm{C}$ se secaron hasta peso constante, aproximadamente $48 \mathrm{~h}$ aproximadamente. Después, las muestras de cada material vegetal se molieron $(<0.1 \mathrm{~mm})$ e identificaron, para luego colocarlas en bolsas de plástico para su análisis en el laboratorio de Nutrición Animal del Departamento de Zootecnia de la Universidad Autónoma Chapingo, Estado de México.

\section{Variables determinadas}

Se determinaron en base seca (\%) con tres repeticiones: proteína cruda (PC), proteína cruda insoluble en detergente neutro (PCIDN), cenizas (Cen), extracto etéreo (EE), fibra detergente neutro (FDN), fibra detergente ácido (FDA), hemicelulosa (Hcel), celulosa (Cel) y lignina (Lig) (AOAC 1990, Van Soest et al. 1991).

\section{Diseño experimental y análisis estadístico}

Se utilizó un diseño experimental completamente al azar, con seis tratamientos (seis gramíneas) y tres repeticiones. Se utilizó la prueba de Tukey para la separación de medias $(p<0.05)$ y las variables por pares se asociaron usando la correlación de Pearson $(p<0.05)$. Para los análisis se empleó el paquete estadístico SPSS (Statistical Package for the Social Sciences por sus siglas en inglés) versión 10.0 (Moreno, 2008).

\section{RESULTADOS}

\section{Pastos guinea y gran-tallo-azul}

Los pastos, guinea (Megathyrsus maximus) y gran-tallo-azul (Andropogon gerardii), mostraron en promedio, los valores más altos $(p<0.05)$ de PC, (11.3\%) y de Cen (10.8\%), con bajos valores de EE
(1.1\%). Por otra parte, los contenidos de FDN, FDA, $\mathrm{Hcel}$, Lig y Cel variaron de intermedios a bajos. Sin embargo, el contenido de PCIDN de guinea fue el más alto $(6.1 \%, p<0.05)$, y el de gran-tallo azul estuvo entre intermedio y bajo (2.1\%), como se observa en las Tablas 1 y 2.

\section{Pastos insurgente y buffel}

Los pastos, insurgente (Urochloa brizantha) y buffel (Andropogon gerardii), mostraron en promedio, $8.0 \%$ de PC, $12.9 \%$ de Cen y valores promedio intermedios a altos de EE (1.5\%). Por otra parte, los contenidos de FDN, Hcel y Lig variaron de intermedios a bajos. Buffel mostró los valores más altos de FDA y Cel e insurgente los más bajos $(p<0.05)$. El contenido de PCIDN de estos pastos varió entre intermedio y bajo (2.1\% en promedio).

\section{Pastos rhodes y llanero}

Los pastos rhodes (Chloris gayana) y llanero (Andropogon gayanus) mostraron en promedio ( $\mathrm{p}<$ $0.05)$, los valores más bajos de PC (4.7\%) y de Cen $(5.7 \%)$ y además mostraron valores promedio intermedios a altos de EE (1.5\%). Por otra parte, los contenidos de FDN, Hcel y Lig variaron de intermedios a altos. De las seis gramíneas evaluadas, buffel mostró los valores más altos de FDA y Cel e insurgente los más bajos $(\mathrm{p}<0.05)$. El contenido de PCIDN de estos pastos varió entre intermedio y bajo (2.1\%), como se observa en las Tablas 1 y 2.

\section{Correlaciones entre variables}

Se encontraron las siguientes correlaciones estadísticamente significativas ( $p<0.05$, Tabla 3 ). La variable PC mostró correlación positiva con las variables Cen y PCIDN y correlación negativa con EE, FDN y Lig. La variable Cen mostró correlación negativa con FDN y Hcel, y el EE mostró correlación positiva y negativa, respectivamente con las variables Lig y PCIDN. La FDN mostró correlación positiva con Hcel. La FDA registró correlación positiva con Cel, y negativa con Hcel y Lig. Las correlaciones entre Cel con Hcel y Lig fueron negativas. 
Tabla 1. Análisis proximal en base seca (\%), de seis gramíneas del estado de Jalisco, México.

\begin{tabular}{lcccc}
\hline Gramínea & $\mathrm{PC}$ & $\mathrm{PCIDN}$ & $\mathrm{Cen}$ & $\mathrm{EE}$ \\
\hline Andropogon gayanus & $4.81^{e}$ & $2.24^{c}$ & $3.57^{e}$ & $1.38^{b}$ \\
Andropogon gerardii & $10.27^{b}$ & $2.06^{c}$ & $7.88^{d}$ & $1.37^{b}$ \\
Cenchrus ciliaris & $7.25^{d}$ & $0.90^{d}$ & $15.24^{a}$ & $1.68^{a}$ \\
Chloris gayana & $4.68^{e}$ & $0.90^{d}$ & $7.73^{d}$ & $1.68^{a}$ \\
Megathyrsus maximus & $12.25^{a}$ & $6.08^{a}$ & $13.64^{b}$ & $0.90^{c}$ \\
Urochloa brizantha & $8.73^{c}$ & $3.30^{b}$ & $10.54^{c}$ & $1.24^{b}$ \\
Error estándar de la media & 0.6689 & 0.4296 & 0.9492 & 0.0669 \\
\hline
\end{tabular}

Medias en la misma columna con distinta literal son estadísticamente diferentes (Tukey, $p<0.05$ ). Cen: Cenizas, PC: Proteína cruda, PCIDN: Proteína cruda insoluble en detergente neutro, EE: Extracto etéreo.

Tabla 2. Análisis de Van Soest en base seca (\%), de seis gramíneas del estado de Jalisco, México.

\begin{tabular}{lccccc}
\hline Especie & FDN & FDA & Hcel & Cel & Lig \\
\hline Andropogon gayanus & $84.49^{a}$ & $49.33^{b}$ & $35.16^{b}$ & $44.31^{b}$ & $4.18^{d}$ \\
Andropogon gerardii & $81.84^{b}$ & $50.46^{b}$ & $31.38^{c}$ & $41.88^{c}$ & $5.23^{c}$ \\
Cenchrus ciliaris & $77.72^{d}$ & $58.03^{a}$ & $19.70^{d}$ & $50.71^{a}$ & $7.07^{b}$ \\
Chloris gayana & $80.84^{b}$ & $41.25^{e}$ & $39.59^{a}$ & $18.09^{e}$ & $23.30^{a}$ \\
Megathyrsus maximus & $78.37^{c d}$ & $47.41^{c}$ & $30.97^{c}$ & $36.14^{d}$ & $5.18^{c d}$ \\
Urochloa brizantha & $78.90^{c}$ & $44.29^{d}$ & $34.61^{b}$ & $36.12^{d}$ & $4.15^{d}$ \\
Error estándar de la media & 0.5699 & 1.2853 & 1.500 & 2.4654 & 1.6577 \\
\hline Medias en la misma columna con distinta literal son estadísticamente diferentes \\
(Tukey, p < 0.05). FDN: Fibra detergente neutro, FDA: Fibra detergente ácido, Hcel: \\
Hemicelulosa, Lig: Lignina, Cel: Celulosa.
\end{tabular}

Tabla 3. Coeficientes de correlación de Pearson entre pares de variables respuesta de seis gramíneas del estado de Jalisco, México.

\begin{tabular}{|c|c|c|c|c|c|c|c|c|c|}
\hline & PC & PCIDN & Cen & EE & FDN & FDA & Hcel & Cel & Lig \\
\hline PC & 1 & $.756^{\star \star}$ & $.541^{\star}$ & $-.756^{\star \star}$ & $-.490^{\star}$ & 0.130 & -0.297 & 0.214 & $-.521^{\star}$ \\
\hline PCIDN & & 1 & 0.275 & $-.954^{\star *}$ & -0.268 & -0.212 & 0.080 & 0.001 & $-.469^{\star}$ \\
\hline Cen & & & 1 & -0.148 & $-.951^{\star *}$ & 0.405 & $-.708^{\star *}$ & 0.230 & -0.131 \\
\hline EE & & & & 1 & 0.125 & 0.187 & -0.113 & -0.070 & $.553^{\star}$ \\
\hline FDN & & & & & 1 & -0.188 & $.540^{\star}$ & -0.034 & 0.012 \\
\hline FDA & & & & & & 1 & $-.928^{\star *}$ & $.892^{\star *}$ & $-.500^{*}$ \\
\hline Hcel & & & & & & & 1 & $-.777^{\star *}$ & 0.433 \\
\hline Cel & & & & & & & & 1 & $-.813^{\star *}$ \\
\hline Lig & & & & & & & & & 1 \\
\hline
\end{tabular}

${ }^{*} p<0.05,{ }^{* *} p<0.01$. PC: Proteína cruda o proteína total, PCIDN: Proteína cruda insoluble en detergente neutro o proteína insoluble, Cen: Cenizas, EE: Extracto etéreo; FDN: Fibra detergente neutro, FDA: Fibra detergente ácido, Hcel: Hemicelulosa, Lig: Lignina, Cel: Celulosa.

\section{DISCUSIÓN}

Los valores altos de PC y de Cen en guinea y gran-tallo-azul (Tabla 1) asociados con valores intermedios a bajos de las variables de la fibra (FDN, FDA, Hcel, Lig y Cel), indican que estas gramíneas podrían recomendarse para la alimentación de rumiantes, desafortunadamente guinea produjo un alto contenido de PCIDN (proteína insoluble), por lo que es más recomendable el pasto gran-tallo-azul por su menor valor de proteína insoluble. Varios valores de las variables determinadas (Tablas 1 y 2 ) se encuen- tran en el rango reportado por distintos investigadores (Rodríguez et al. 2013, Ziehr et al. 2014, OrtegaAguirre et al. 2015, Valles de la Mora et al. 2016, Beltrán et al. 2017, Merlo et al. 2017, Almaraz-Buendía et al. 2019, Barragán y Cajas 2019, López-Vigoa et al. 2019).

Las correlaciones negativas entre PC con EE, FDN y Lig implican que las gramíneas con menor FDN como guinea y gran-tallo-azul tendrán más PC, y mayor contenido celular (CC): 100\% - FDN, y probablemente la formación de PC es a expensas 
de la disminución de EE y de Lig. El razonamiento aquí es que, tanto en este estudio como en la literatura consultada, valores altos de PC se observan asociados con valores altos de Cen, e inversamente. Por ejemplo, guinea y gran-tallo-azul fueron altos en PC y altos en Cen, mientras que rhodes y llanero fueron bajos en PC y bajos en Cen (Tabla 1). Al respecto, Almaraz-Buendía et al. (2019), encontraron que a valores altos de Cen, presentan valores altos de PC. Esta correlación positiva entre Cen y PC implica que conociendo el contenido de Cen se puede tener una idea del contenido de PC. Sobre lo mismo Ortega-Aguirre et al. (2015) reportan que gramíneas con altos valores de PC mostraron valores altos de Cen, mientras que Garay-Martínez et al. (2020) que bajos valores de Lig se correlacionan con altos valores de PC. Además, valores altos de PC se encuentran asociados con valores bajos de Lig (Tablas 1 y 2). Por ejemplo, guinea y grantallo-azul que mostraron altos valores de PC tuvieron valores intermedios a bajos de FDN y EE, y bajos de Lig. Al respecto Garay-Martínez et al. (2020) reportan que valores altos de Cen estuvieron asociados con valores bajos de FDN. Es por ello que en este estudio se observó (Tabla 3) que a mayor contenido de Cen, mayor contenido de PC (correlación positiva que puede indicar una necesidad de fertilización nitrogenada para que aumente la PC de los pastos bajo estudio).

Además, se observaron correlaciones negativas entre Cen y FDN, entre Cen y Hcel, entre Lig y PC y entre Lig y PCIDN (Tabla 3). Concordante, en este estudio (Tablas 1 y 2 ), los pastos buffel, guinea e insurgente produjeron altos valores de Cen asociados con bajos valores de FDN e intermedios a bajos de Hcel, mientras que gran-tallo-azul y guinea mostraron altos valores de Cen, asociados con valores intermedios a bajos de Lig. Adicionalmente, con guinea, insurgente y rhodes (Tabla 1), se observa que, a mayores valores de $\mathrm{PC}$, hay mayores valores de PCIDN. Las correlaciones negativas se explican porque a mayor proporción de tallos, menor proporción de hojas y las hojas tienen más PC (Koutsoukis et al. 2016) y menos FDN que los tallos. En consecuencia, al compararlo con rhodes, guinea mostró mayor valor de PC y menor de FDN, debido probablemente a su mayor proporción de hojas y menor proporción de tallos con respecto a rhodes. Sin embargo, gran-tallo-azul produjo menor proteína insoluble que guinea. Lo cual probablemente se debió a que este pasto desvía su proteína hacia las hojas y no hacia el tallo, cuya proteína es más insoluble por su mayor contenido de compuestos lignocelulósicos.

\section{CONCLUSIONES}

Los pastos guinea y gran-tallo-azul, defoliados a 35 días después del rebrote, presentaron los valores más altos de proteína cruda y porcentajes bajos de extracto etéreo y de lignina; el pasto guinea mostró bajo porcentaje de proteína insoluble. Por tanto, el mejor contenido nutricional lo produjo el pasto gran-tallo-azul (Andropogon gerardii).

\section{LITERATURA CITADA}

Almaraz-Buendía I, García AM, Sánchez SP, Torres SN, Herrera PJ, Bottini LMB, Rojas GAR (2019) Análisis bromatológico y producción de gas in vitro de forrajes utilizados en el trópico seco mexicano. Archivos de Zootecnia 68: 260-266.

AOAC (1990) Official methods of analysis. Association of Official Analytical Chemists. 15th Ed. Arlington, Virginia, USA. 771p.

Barragán HWA, Cajas GYS (2019) Cambios bromatológicos y estructurales en Megathyrsus maximus bajo cuatro arreglos silvopastoriles. Ciencia y Tecnología Agropecuaria 20: 231-244.

Beltrán LS, García DCA, Loredo OC, Urrutia MJ, Hernández AJA, Gámez VHG (2017) "Titán” y "Regio", variedades de pasto Buffel (Pennisetum ciliare) (L.) Link para zonas áridas y semiáridas. Revista Mexicana de 
Ciencias Pecuarias 8: 291-295.

Castrejón PFA, Corona GL, Rosiles MR, Martínez PP, Lorenzana MAV, Arzate VLG, Olivos AP, Guzmán AP, García PA, Avilés NJN, et al. (2017) Características nutrimentales de gramíneas, leguminosas y algunas arbóreas forrajeras del trópico mexicano: fracciones de proteína (A, B1, B2, B3 y C), carbohidratos y digestibilidad In vitro. 1a Ed. Facultad de Medicina Veterinaria y Zootecnia, UNAM. México. 172p.

Daba AW, Qureshi AS, Nisaren BN (2019) Evaluation of some rhodes grass (Chloris gayana) genotypes for their salt tolerance, biomass yield and nutrient composition. Applied Sciences 9: 143. DOI: 10.3390/app9010143.

Enríquez QJF, Meléndez NF, Bolaños AED, Esqueda EVA (2011) Producción y manejo de forrajes tropicales. Imprenta Alfa y Omega. INIFAP. Veracruz, México. 443p.

Fernández TL, Castillo GE, Ocaña ZE, Valles de la MB, Jarillo RJ (2006) Características de la vegetación en gramas nativas solas o asociadas con Arachis pintoi CIAT 17434 en pastoreo rotacional intensivo. Técnica Pecuaria en México 44: 365-378.

Garay-Martínez JR, Estrada-Drouaillet B, Bautista-Martínez Y, Bernal-Flores Á, Mendoza-Pedroza SI, MartínezGonzález JC, Sosa-Montes E, Joaquín-Cancino S (2020) Forage yield and quality of buffel 'H-17' and Urochloa hybrids at different regrowth ages under semi-arid conditions. Grassland Sience 66: 277-284

IIEG-Jalisco (2018) Tonaya Diagnostico del municipio. Instituto de Información, Estadística y Geográfica del Estado de Jalisco. https://iieg.gob.mx/contenido/Municipios/Tonaya.pdf. Fecha de consulta: 10 de septiembre de 2020.

Kupryś-Caruk M, Podlaski S, Kotyrba D (2019) Influence of double-cut harvest system on biomass yield, quality and biogas production from C4 perennial grasses. Biomass and Bioenergy 130: 105376. DOI: 10.1016/j. biombioe.2019.105376.

Koutsoukis C, Akrida-Demertzi K, Demertzis PG, Roukos C, Voidarou C, Kandrelis S (2016) The variation of crude protein and total fat of the main grassland plants, in various stages of growth, in "Kostilata" Subalpine Grassland in Theodoriana, Arta, Greece. Ekin Journal of Crop Breeding and Genetics 2: 69-75.

López-Vigoa O, Lamela-López L, Sánchez-Santana T, Olivera-Castro Y, García-López R, Herrera-Villafranca M, González-Ronquillo M (2019) Evaluación del valor nutricional de los forrajes en un sistema silvopastoril. Pastos y Forrajes 42: 57-67.

Merlo MFE, Ramírez AL, Ayala BAJ, Ku VJC (2017) Efecto de la edad de corte y la época del año sobre el rendimiento y calidad de Brachiaria brizantha (A. Rich.) Staff en Yucatán, México. Journal of the Selva Andina Animal Science 4: 116-127.

Moreno GE (2008) Manual de uso de SPSS. 1a Ed. Universidad Nacional de Educación a Distancia. Madrid, España. 267p. http://e-spacio.uned.es/fez/eserv/bibliuned:500727/Guia_SPSS.pdf

Ortega-Aguirre CA, Lemus FC, Bugarín PJO, Alejo SG, Ramos QA, Grageola NO, Bonilla CJA (2015) Características agronómicas, composición, bromatología. Digestibilidad y consumo animal en cuatro especies de pastos de los géneros Brachiaria y Panicum. Tropical and Subtropical Agroecosystems. 18: 291-301.

Peña PM, Del Pozo P (2004) Explotación de pastos y forrajes tomo II. Editorial Félix Varela. Habana, Cuba. $241 p$.

Rodríguez RMR, González SA, Yáñez MA, Silva LM, Gómez ECI (2013) Composición química de recursos forrajeros para la alimentación de ovinos en Colima. INIFAP, CIRPAC. Campo Experimental Tecomán. Folleto técnico No.3. Tecomán, Colima, México. 59p. 
Valles de la Mora B, Castillo GE, Bernal BH (2016) Rendimiento y degradabilidad ruminal de materia seca y energía de diez pastos tropicales cosechados a cuatro edades. Revista Mexicana de Ciencias Pecuarias 7: $141-158$.

Van Soest PJ, Robertson JB, Lewis BA (1991) Methods for dietary fiber, neutral detergent fiber, and nonstarch polysacaccharides in relation to animal nutrition. Journal of Dairy Science 74: 3583-3597.

Villegas DG, Bolaños MA, Olguín PL (2001) La ganadería en México. I.5.1. Editorial Plaza y Valdés. México. $158 p$.

Ziehr RD, Rea GL, Douglas JL, Spaeth KE, Peacock GL, Muir JP (2014) Ontogenesis and nutritive value of warm-season perennial bunch grasses. Tropical Grasslands-Forrajes Tropicales 2: 188-196. 